\title{
Investigation of energy stored in copper processed by combination of SPD methods
}

\author{
A.A. Gimazov ${ }^{1, \uparrow}$, A.P. Zhilyaev ${ }^{2,3}$ \\ †Gimazov@gmail.com \\ ${ }^{1}$ LLC «BashNIPIneft», 86/1 Lenina street, 450039, Ufa, Russia \\ ${ }^{2}$ Fundació CTM Centre Tecnològic, Plaça de la Ciencia 2, Manresa, Barcelona, 08242, Spain \\ ${ }^{3}$ Institute for Metals Superplasticity Problems of Russian Academy of Science, 39 Khalturina, 450001, Ufa, Russia
}

\begin{abstract}
A calorimetric study of commercial pure copper processed by combination of severe plastic deformation methods - highpressure torsion, equal channel angular pressing and machining was performed. Calculated activation energy and enthalpy for the three peaks observed in the curves of differential scanning calorimetry defined relaxation processes corresponding to these peaks: the first peak - redistribution dislocations without the formation of new grain boundaries, the second peak - redistribution dislocations with partial annihilation and formation of low-angle grain boundaries, the third peak recrystallization. Comparative analysis showed that the differential scanning calorimetry curves for samples subjected to high-pressure torsion deformation and sequencing methods of equal-channel angular pressing and high-pressure torsion showed no presence of the second peak. For samples in which the processing method involved machining, second peak detected steadily and is characterized by high values of enthalpy. It is shown that the difference in the curves for the various combinations of methods of intensive plastic deformation associated with substantially different processing speed, which leads to increased twins concentration in deformed material that impede cross slip migration of dislocation, thereby contributing to their accumulation in the material and prevent redistribution on the early stages of heating. Increased concentration of dislocation is used in the process of forming a microstructure that provides a structure with a smaller average grain size, which leads to an increase in the microhardness of the high-speed deformed materials compared with the samples slowly deformed.
\end{abstract}

Keywords: severe plastic deformation, differential calorimetry, pure metal, copper.

\section{Исследование энергии, запасенной в меди при обработке} комбинацией методов интенсивной пластической деформации

\author{
Гимазов А.А. ${ }^{1, \dagger}$, Жиляев А.П. ${ }^{2,3}$ \\ †Gimazov@gmail.com \\ ${ }^{1}$ ООО «БашНИПИнефть», ул.Ленина 86/1, 450039, Уфа, Россия \\ ${ }^{2}$ Fundació CTM Centre Tecnològic, Plaça de la Ciencia 2, Manresa, Barcelona, 08242, Spain \\ ${ }^{3}$ Институт проблем сверхпластичности металлов РАН, ул. Халтурина 39, 450001, Уфа, Россия
}

Проведено калориметрическое исследование технически чистой меди, подвергнутой обработке комбинацией методов интенсивной пластической деформации - кручение под высоким давлением, равноканальное угловое прессование и высокоскоростная резка. Рассчитана энергия активации и энтальпия для трех пиков, наблюдаемых на кривых дифференциальной сканирующей калориметрии, определены релаксационные процессы, соответствующие данным пикам: первый пик - перераспределение дислокаций без образования новых границ зерен, второй пик - перераспределение дислокаций с частичной аннигиляцией и образованием малоугловых границ зерен, третий пик - рекристаллизация. Сравнительный анализ показал, что кривые дифференциальной сканирующей калориметрии для образцов чистой меди, подвергнутых деформацией кручением под высоким давлением и последовательности методов равноканального углового прессования и кручения под высоким давлением, не демонстрируют наличие второго пика, либо данный пик крайне мал для его уверенной аттестации. Для образцов, в обработке которых задействован метод высокоскоростной резки, пик, соответствующий перераспределению дислокаций с аннигиляцией и образованием малоугловых границ, выявляется уверенно и характеризуется высокими значениями энтальпии. Показано, что отличия в кривых для различных комбинаций методов интенсивной пластической деформации связаны с суще- 
ственно различной скоростью обработки, что приводит к повышенной концентрации двойников в деформированном материале, которые затрудняют поперечное скольжение дислокаций, тем самым способствуют их накоплению в материале и препятствуют перераспределению на ранних стадиях нагревания. Повышенная концентрация дислокаций используется в процессе формирования микроструктуры, что позволяет получить структуру с меньшим средним размером зерна, что приводит к повышению микротвердости материала, обработанного с использованием высокоскоростной обработки по сравнению с медленно деформированными образцами.

Ключевые слова: интенсивная пластическая деформация, дифференциальная калориметрия, чистый металл, медь.

\section{1. Введение}

Интенсивная пластическая деформация (ИПД) позволяет получать массивные образцы, характеризующиеся ультрамелкозернистой (УМЗ) и нано структурой. В настоящее время разработано множество способов ИПД: многократная прокатка [1], равноканальное угловое прессование (РКУП) [2, 3], кручение под высоким давлением (КВД) [4], высокоскоростная резка (ВСР) [5], а также опробовано множество модификаций данных методов.

Активный интерес к этим методам обусловлен необычными физическими [6] и механическими [7-10] свойствами материалов, подвергнутых упомянутым обработкам.

Существование принципиально различающихся методов ИПД позволяет использовать их последовательно. В работах $[11,12]$ описаны примеры того, что возможно достижение заметного сдвига точки насыщения в область больших степеней деформации с соответствующим более длинным периодом эффективного воздействия на структуру материала. Несмотря на большое количество работ, посвященных эволюции микроструктуры и свойств металлов и сплавов высокими степенями ИПд, очень малое количество исследований посвящено изучению материалов, деформированных комбинацией методов ИПД.

Основным источником полезных свойств УМЗ и нано кристаллических материалов являются большеугловые границы зерен (БУГ), также они являются наиболее стабильными дефектами структуры, в то время как вакансии и дислокации, которые также запасают часть энергии пластической деформации, являются своего рода строительным материалом для БУГ и начинают релаксировать при более низких температурах. В зарубежных и отечественных публикациях приводятся данные дифференциальной сканирующей калориметрии (ДСК), демонстрирующие, что при различных методах ИПД концентрация дефектов кристаллической решетки (вакансий, дислокаций и границ зерен) отличаются; для комбинации методов подобных исследований не проводилось. ДСК позволяет понять, как запасена энергия деформации в СМК и нано-кристаллическом материале. Понимание необходимо как с точки зрения определения потенциала примененного метода ИПД, так и с точки зрения использования полученных материалов в практических целях.

\section{2. Материал и методики}

Для изучения запасенной энергии в материале, полученном последовательностью методов ИПД, целесообразно выбрать чистый однофазный металл, чтобы исключить влияние фазовых переходов при ИПД, что было зафиксировано для чистого циркония [13], и нагреве, например, медь, обладающую гранецентрированной кубической (ГЦК) кристаллической решеткой. Степень чистоты исследуемого металла 99.9\% (технически чистая медь).

В работе использовались 3 вида ИПД: РКУП (4 прохода по маршруту ВС на установке с $\left.\Phi=90^{\circ}, \Psi=0^{\circ}\right)$, КВД (на наковальне Бриджмена, 5 оборотов под давлением 6 ГПа) и ВСР (со скоростью 30 м/мин, резцом с рабочим углом $\alpha=40^{\circ}$ ). Все виды деформации проводились при комнатной температуре.

Для исследования было подготовлено 4 образца меди: 1) деформированный КВД; 2) ВСР с последующим компактированием стружки методом КВД; 3) РКУП с последующей обработкой методом КВД; 4) РКУП с последующей ВСР полученных прутков, с последующим компактированием стружки методом КВД; для краткости образцы обозначаются КВД, ВСР+КВД, РКУП+КВД и РКУП+ВСР+КВД соответственно. Образцы после компактирования представляли собой гладкие беспористые диски без видимых невооруженным глазом и в оптический микроскоп включений. Полученные образцы имели форму диска диаметром 9 мм и толщиной 0.3-0.5 мм. Рентгеноструктурный анализ не выявил в данных образцах присутствия оксидов. Расчет степени деформации проводился согласно $[14,15,16]$. Для последовательности методов степень деформации приравнивалась алгебраической сумме степеней деформации, достигнутых для каждого из использованных методов.

Дифференциальная сканирующая калориметрия (ДСК) была выполнена с использованием прибopa Perkin-Elmer DSC 7 в университете Autònoma de Barcelona. Измерения проводились при нагреве со скоростью 10, 20, 40 и $80 \mathrm{~K} /$ мин и охлаждении со скоростью около 120 К/мин в атмосфере аргона. Каждый образец был разделен на несколько кусочков для проведения ДСК с разной скоростью. Калориметрия проводилась в два этапа: сначала в ходе нагрева с определенной скоростью до температуры $600^{\circ} \mathrm{C}$ записывалась кривая поглощения, затем образец охлаждался со скоростью 120 К/мин, затем повторно проводился нагрев того же образца с той же скоростью для записи базовой линии. Для анализа использовалась разница между линией поглощения и базовой линией. 


\section{3. Результат и обсуждение}

На рис. 1 приведены кривые ДСК для образца РКУП+ВСР+КВД. На данных кривых можно различить 3 экзотермических пика. Однако, чаще всего, пики, соответствующие различным процессам, значительно уширяются и накладываются друг на друга, поэтому не всегда удается их четко разделить, что значительно осложняет определение энергии активации каждого из процессов и соответствующей энтальпии. Для уточнения положения центров пиков была применена следующая методика - строилась синтетическая кривая (жирная кривая), являющаяся алгебраической суммой трех Гауссовых пиков (тонкие кривые), параметры каждого из пиков подбирались таким образом, чтобы их суммарная синтетическая кривая была максимально похожа на экспериментальную ДСК кривую. Полученные центры пиков приведены в таблице 1.
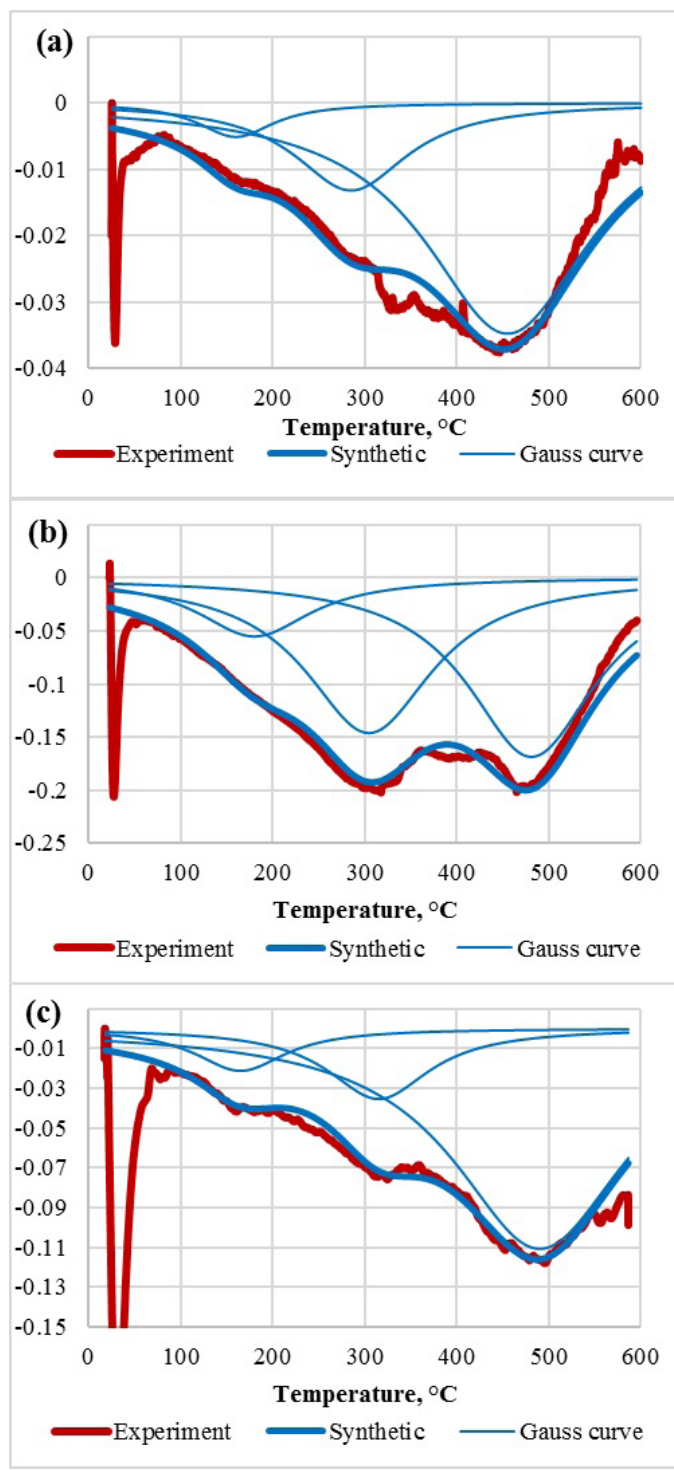

Pис. 1. (Color online) Кривые ДСК для скорости нагрева (a) $20 \mathrm{~K} / \mathrm{мин,} \mathrm{(b)} 40 \mathrm{~K} / \mathrm{мин,} \mathrm{(c)} 80 \mathrm{~K} / \mathrm{мин}$

Fig. 1. (Color online) DCS curves for heating rates (a) $20 \mathrm{~K} / \mathrm{min}$, (b) $40 \mathrm{~K} / \mathrm{min}$, (c) $80 \mathrm{~K} / \mathrm{min}$.
Табл. 1. Центры пиков и энергия активации для образцов меди после ИПД.

Table 1. Peaks positions and activation energy for copper samples after SPD.

\begin{tabular}{|c|c|c|c|c|c|c|c|c|c|c|c|c|}
\hline Параметр & \multicolumn{4}{|c|}{ Пик 1} & \multicolumn{4}{|c|}{ Пик 2} & \multicolumn{4}{|c|}{ Пик 3} \\
\hline Parameter & \multicolumn{4}{|c|}{ Peak 1} & \multicolumn{4}{|c|}{ Peak 2} & \multicolumn{4}{|c|}{ Peak 3} \\
\hline $\begin{array}{c}\text { Скорость } \\
\text { нагрева } b \text {, } \\
\text { К/мин }\end{array}$ & \multirow[t]{2}{*}{10} & \multirow[t]{2}{*}{20} & \multirow[t]{2}{*}{40} & \multirow[t]{2}{*}{80} & \multirow[t]{2}{*}{10} & \multirow[t]{2}{*}{20} & \multirow[t]{2}{*}{40} & \multirow[t]{2}{*}{80} & \multirow[t]{2}{*}{10} & \multirow[t]{2}{*}{20} & \multirow[t]{2}{*}{40} & \multirow[t]{2}{*}{80} \\
\hline $\begin{array}{c}\text { Heat rate } b \text {, } \\
\mathrm{K} / \mathrm{min}\end{array}$ & & & & & & & & & & & & \\
\hline \multicolumn{13}{|c|}{ КВД } \\
\hline \multicolumn{13}{|c|}{ HPT } \\
\hline Пик, ${ }^{\circ} \mathrm{C}$ & \multirow{2}{*}{190} & \multirow{2}{*}{200} & \multirow{2}{*}{217} & \multirow{2}{*}{-} & & \multirow{2}{*}{-} & \multirow{2}{*}{-} & & \multirow{2}{*}{452} & \multirow{2}{*}{471} & & \multirow{2}{*}{-} \\
\hline Peak, ${ }^{\circ} \mathrm{C}$ & & & & & - & & & - & & & 483 & \\
\hline $\begin{array}{c}E_{a}, \\
\text { КДж/моль } \\
\text { (эВ/атом) }\end{array}$ & \multirow{2}{*}{\multicolumn{4}{|c|}{$87.1(0.90)$}} & \multirow{2}{*}{\multicolumn{4}{|c|}{-}} & \multirow{2}{*}{\multicolumn{4}{|c|}{$187.4(1.94)$}} \\
\hline $\begin{array}{l}E_{a}, \mathrm{~kJ} / \mathrm{mol} \\
(\mathrm{eV} / \text { atom })\end{array}$ & & & & & & & & & & & & \\
\hline \multicolumn{13}{|c|}{ РКУП+ВСР+КВД } \\
\hline \multicolumn{13}{|c|}{$\mathrm{ECAP}+$ Machining $+\mathrm{HPT}$} \\
\hline Пик, ${ }^{\circ} \mathrm{C}$ & \multirow{2}{*}{-} & & & & & & & & & & & \\
\hline Peak, ${ }^{\circ} \mathrm{C}$ & & 100 & 180 & 191 & - & 285 & 300 & 315 & - & 450 & 480 & 490 \\
\hline $\begin{array}{c}E_{a}, \\
\text { кДж/моль } \\
\text { (эВ/атом) }\end{array}$ & & 64.7( & $0.67)$ & & & 16.5 & $(1.21$ & & & 65.1 & $(1.7$ & \\
\hline $\begin{array}{l}E_{a}, \mathrm{~kJ} / \mathrm{mol} \\
(\mathrm{eV} / \text { atom })\end{array}$ & & & & & & & & & & & & \\
\hline
\end{tabular}

Энергия активации определялась методом Киссингеpa [17] с помощью соотношения:

$$
\ln \left(\frac{b}{T^{2}}\right) \cong-\frac{E_{a}}{R T}+\ln \left(\frac{R K_{0}}{E_{a}}\right),
$$

где $b$ - скорость нагрева, $T$ - температура пика, $E_{a}-$ энергия активации процесса, соответствующего данному пику, $R-$ универсальная газовая постоянная. Метод состоит в нахождении коэффициента наклона прямой аппроксимирующей данные для различных скоростей нагрева в координатах $\ln \left(b / T^{2}\right)$ от $1 / T-$ данная величина представляет собой значение энергии активации соответствующего процесса, отнесенное к величине универсальной газовой постоянной $E_{a} / R$. Экспериментальные точки и аппроксимирующие их прямые приведены на рис. 2, полученные таким образом значения энергии активации - в таблице 1. 
ДСК кривые для образца меди после КВД анализировались аналогичным способом, результаты даны в таблице 1. Для образца после КВД положение второго пика определялось с большой погрешностью, поэтому энергия активации для него не вычислена. Для меди после ВСР+КВД лишь одна кривая позволяет различать пики, данная кривая будет использована при качественном анализе. На рисунке 3 приведены для качественного сравнения ДСК кривые для образцов после КВД, ВСР+КВД и РКУП+КВД, полученные на скорости 20 К/мин. Сравнение данных кривых выявляет особенность ДСК кривой для РКУП+КВД и КВД образцов на месте 2го пика в области $300{ }^{\circ} \mathrm{C}$ наблюдается плато, энтальпия для данного процесса составляет крайне малое значение (табл. 2).

Согласно Ван-Бюрену [18] для меди энергия активации первого, меньшего по температуре, пика, соответствует процессу перераспределения дислокаций без образования новых границ (0.7 эВ/атом), второго пика - процессу перераспределения дислокаций с частичной аннигиляцией и образованием МУГ (1.2 эВ/атом), третий пик, по всей видимости, должен соответствовать процессу рекристаллизации. Меньшая величина энергии активации для него, по сравнению с определенной Ван-Бюреном (2 эВ/атом), может быть обусловлена УМЗ состоянием образцов меди после ИПД. Отсутствие второго пика, может быть связано с высокой долей МУГ в образце после КВД - в работе [19] методом EBSD OIM показано, что доля МУГ в КВД и РКУП+КВД образцах составляет $32 \%$ и $35 \%$ соответственно, тогда как после ВСР+КВД и РКУП+ВСР+КВД эта доля составляет $19 \%$ и $25 \%$.

Сравнение положения пиков и величин энергии активации показывает, для КВД пики смещаются в сторону больших температур. Возможно, пик, соответствующий образованию МУГ, подвержен данной тенденции сильнее, то есть сильнее смещен в область повышенных температур и сливается с пиком, соответствующим рекристаллизации. В пользу данного предположения говорит и вид третьего пика $3-$ он похож на суперпозицию 2 пиков.

Для проверки данной гипотезы третий пик для всех ДСК кривых полученных на образце после КВД был подобран в виде суммы 2 кривых Гаусса, это позволило уточнить центр пиков и рассчитать энергию активации для каждого из них. Величина энергии активации составила для обоих величину близкую к 180 кДж/моль, что является слишком высоким значением для образования МУГ. Таким образом, гипотеза о смещении пика образования МУГ в область больших температур не является состоятельной.

По всей видимости, вследствие высокой плотности границ зерен в материале, в образцах после КВД и РКУП+КВД большая часть дислокаций в ходе процесса перераспределения дислокаций (соответствующей первому пику) выходит на поверхность зерен и в диапазоне от $100^{\circ} \mathrm{C}$ до $300^{\circ} \mathrm{C}$ наблюдается только один пик, так как в образовании МУГ участвует малое количество дефектов. Для КВД один пик наблюдали в работах $[20,21]$ для РКУП в работе [22]. Значения энергии

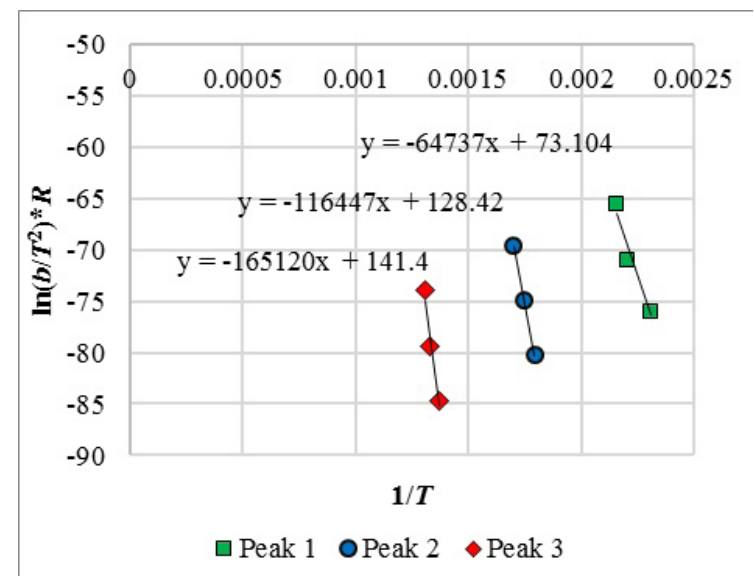

Рис. 2. График Киссингера для образца РКУП+ВСР+КВД. Fig. 2. Kissinger plot for ECAP+Machining+HPT sample.

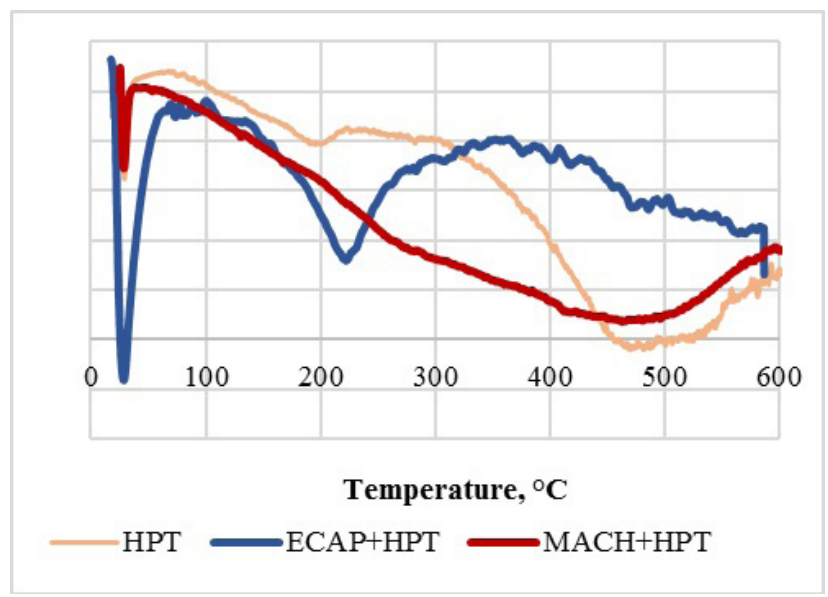

Pис. 3. (Color online) ДСК кривые 20 К/мин для образцов меди после ИПД.

Fig. 3. (Color online) $20 \mathrm{~K} / \mathrm{min}$ DSC curves for copper samples after SPD.

Табл. 2. Значения энтальпии для меди после ИПД. Table 2. Enthalpy for copper after SPD.

\begin{tabular}{|c|c|c|c|c|c|}
\hline \multirow{2}{*}{$\begin{array}{c}\text { Метод } \\
\text { ИПд }\end{array}$} & \multicolumn{4}{|c|}{ Энтальпия, Дж/г } & \multirow{2}{*}{$\begin{array}{c}\text { Степень дефор- } \\
\text { мации }\end{array}$} \\
\hline & \multicolumn{4}{|c|}{ Enthalpy, J/g } & \\
\hline \multirow{2}{*}{$\begin{array}{c}\text { SPD } \\
\text { method }\end{array}$} & Пик 1 & Пик 2 & Пик 3 & Сумма & \multirow{2}{*}{$\begin{array}{c}\text { Deformation } \\
\text { level }\end{array}$} \\
\hline & Peak 1 & Peak 2 & Peak 3 & Summ & \\
\hline КВД & \multirow{2}{*}{0.78} & \multirow{2}{*}{0.09} & \multirow{2}{*}{6.64} & \multirow{2}{*}{7.51} & \multirow{2}{*}{7.5} \\
\hline HPT & & & & & \\
\hline $\begin{array}{l}\text { ВСР+ } \\
\text { КВД }\end{array}$ & \multirow{2}{*}{2.92} & \multirow{2}{*}{5.74} & \multirow{2}{*}{8.91} & \multirow{2}{*}{17.57} & \multirow{2}{*}{8.5} \\
\hline $\begin{array}{c}\text { Mach+ } \\
\text { HPT }\end{array}$ & & & & & \\
\hline $\begin{array}{c}\text { РКУП+ } \\
\text { ВСР+ } \\
\text { КВД }\end{array}$ & \multirow{2}{*}{1.02} & \multirow{2}{*}{5.48} & \multirow{2}{*}{9.21} & \multirow{2}{*}{15.71} & \multirow{2}{*}{10.9} \\
\hline $\begin{array}{c}\text { ECAP+ } \\
\text { Mach+ } \\
\text { HPT }\end{array}$ & & & & & \\
\hline
\end{tabular}


активации для данного пика были определены равными 77 кДж/моль, накопленной энтальпии для 4-16 проходов 0.95 Дж/г [22], что близко к результатам полученными в данной работе. Исследование меди в области высоких температур проведено в работе [23] и показано наличие пика на кривой ДСК в области $450^{\circ} \mathrm{C}$. Энергия активации определенная для процесса, соответствующего данному пику, составила $~ 0.96$ эВ/атом, накопленная энтальпия 0.92 Дж/г - величины заметно меньшие, полученных в данной работе. Отличие может быть связано с более высокой чистотой изучаемых в [23] образцов $(99.995 \%)$ и иным способом деформации - КВД 6 оборотов под давлением 2.2 ГПа, полученный образец представлял собой цилиндр диаметром 30 мм и высотой 7 мм.

Для образцов ВСР+КВД и РКУП+ВСР+КВД процесс релаксации идет иначе - большая часть дислокаций участвует в процессе образования МУГ, что позволяет нам видеть второй пик на кривой ДСК, и суммарная энтальпия для таких образцов заметно выше, чем для КВД. В виде механических свойств и микроструктуры это выражается в меньшем, по сравнению с КВД, средним размером зерна и большей микротвердостью [24]. То есть существует дополнительный фактор, препятствующий движению дислокаций и их выходу в ГЗ на ранних стадиях нагревания. При малых скоростях деформации (и при комнатной и выше температурах) имеет место дислокационный механизм деформации, когда формирование структуры контролируется образованием, движением и перегруппировкой дислокаций. В работе [25] авторы показывают, что при высоких скоростях и низких температурах, когда параметр $\ln Z$ (где $Z=\mathrm{d} \varepsilon / \mathrm{d} t^{*} \exp (Q / R T)$ - параметр Зенера-Холомона, $\mathrm{d} \varepsilon / \mathrm{d} t-$ скорость деформации, $Q-$ энергия активации зернограничной диффузии, $R-$ универсальная газовая постоянная, $T$ - температура деформации) превышает значение $~ 30$, начинает действовать также и двойниковый механизм деформации, который заключается в том, что изначально исходные зерна преобразуются в набор тонких пластин, разделенных двойниковыми границами, и при последующей деформации они преобразуются сначала в удлиненные зерна, а затем в равноосные. Очевидно, что в образцах, деформированных с высокой скоростью, плотность двойников будет повышенной по сравнению с медленнодеформированными образцами, что подтверждается микроскопическими исследованиями даже для алюминия, обладающего высоким значением энергии дефектов упаковки [26].

Используя значение $Q=72.5$ кДж/моль и скорости деформации $10^{-1}$ (для РКУП и КВД) и $10^{3}$ (для ВСР), можно рассчитать $\ln Z$, который составляет 27 и 37 соответственно, что говорит о повышенной, по сравнению с КВД, плотности двойников в образцах, в обработке которых использован метод ВСР. Наличие двойников затрудняет поперечное скольжение дислокаций, способствуя их накоплению [13]. Таким образом, в материале, обработанном методом скоростной деформации, накапливается большее количество дислокаций, чем в медленнодеформированном. Кроме того, накопленные дислокации сохраняются в материале при нагреве до более высоких температур и используются при формировании микроструктуры, о чем говорит значительное превышение накопленной энтальпии для образцов ВСР+КВД и РКУП+ВСР+КВД по сравнению с КВД.

\section{4. Заключение}

Анализ кривых ДСК показал, что в диапазоне от $100^{\circ} \mathrm{C}$ до $600^{\circ} \mathrm{C}$ можно наблюдать 3 пика соответствующих: 1) перераспределению дислокаций без образования МУГ; 2) аннигиляции дислокаций и образованию МУГ; 3) рекристаллизации.

Повышенное содержание двойников в материалах, полученных деформацией с высокой скоростью, по сравнению с медленно деформированными, позволяет накапливать большее количество дислокаций и использовать их при формировании микроструктуры, что позволяет накапливать в материале больше энергии пластической деформации.

\section{Литература/References}

1. G.P. Dinda, H. Rösner, G. Wilde. Mater. Sci. Eng. A. 410 - 411, 328-331 (2005)

2. R.Z. Valiev, R.K. Islamgaliev, I. V. Alexandrov. Prog. Mater. Sci. 5 (2), 103 - 189 (2000)

3. R.Z. Valiev, T.G. Langdon. Prog. Mater. Sci. 51 (7), $881-981$ (2006)

4. A.P. Zhilyaev, T. G. Langdon. Prog. Mater. Sci. 53 (6), $893-979$ (2008)

5. S. Swaminathan, T.L. Brown, S. Chandrasekar, T. R. McNelley, W.D. Compton. Scripta Mater. 56 (12), 1047 - 1050 (2007)

6. E. A. Korznikova, S.Y. Mironov, A.V. Korznikov, A.P. Zhilyaev, T.G. Langdon. Materials Science and Engineering: A. 556. $437-445$ (2012).

7. S. Zhang, Y.C. Wang, S. Li, A. P. Zhilyaev, E. Korznikova, G. I. Raab, T.G. Langdon Materials Science and Engineering: 641, 29-36 (2015).

8. S. Zhang, Y.C. Wang, S. Li, A. P. Zhilyaev, E. Korznikova, G.I. Raab, T.G. Langdon Materials Science and Engineering: 645, $311-317$ (2015).

9. L. Wang, Y.C. Wang, S.K. Li, A.P. Zhilyaev, A. V. Korznikov, E. Korznikova, T. G. Langdon. Scripta Materialia. 77, 33 - 36 (2014).

10. S. Zhang, Y.C. Wang, S. Li, A. P. Zhilyaev, E. Korznikova, D. V. Gunderov, G.I. Raab, T.G. Langdon Materials Science and Engineering: A. 634, 64-70 (2015).

11. N. Lugo, N. Llorca, J. M. Cabrera, Z. Horita. Mater. Sci. eng. A. 477 (1-2), $366-371$ (2008)

12. A.P. Zhilyaev, J. Gubicza, G. Nurislamova, Á. Révész, S. Suriñach, M. D. Baró, T. Ungár. Phys. Stat. Sol. (a). 198 (2), $263-271$ (2003)

13. M.T. Perez-Prado, A.A. Gimazov, O.A. Ruano, M. E Kassner. and A.P. Zhilyaev. Scripta Mater. 58, 219-222 (2008)

14. H. S. Kim. J. Mater. Process. Techn. 113, 617-621 (2001)

15. T. Aida, K. Matsuki, Z. Horita, T. G. Langdon. Scripta Mater. 44, 575 - 579 (2001) 
16. S. Swaminathan, T.L. Brown, S. Chandrasekar, T. R. McNelley, W.D. Compton. Scripta Mater. 56 (12), 1047 - 1050 (2007).

17. H.E. Kissinger. Anal. Chem. 29 (11), 1702 - 1706 (1957)

18. S.S. Gorelik, S.V. Dobatkin, L. M Kaputkina. Recrystallization of metals and alloys. Moscow, MISIS. (2005) 432 p. (in Russian) [С. С. Горелик, С. В. Добаткин, Л. М. Капуткина. Рекристаллизация металлов и сплавов. Москва, МИСИС, 2005. 432с]

19. A.P. Zhilyaev, S. Swaminathan, A.A. Gimazov, T. R. McNelley, T. G. Langdon. J Mater Sci. 43, 7451 - 7456 (2008) DOI:10.1007/s10853-008-2714-y

20. E. A. Korznikova. Letters on Materials. 2, 67 - 70 (2012). (in Russian) [Е. А. Корзникова. Письма о материалах. 2, $67-70$ (2012)]

21. D. Setman, E. Schafler, E. Korznikova, M. J. Zehetbauer. Mater. Sci. Eng. A. 493, 116-122 (2008)

22. W. Q. Cao, C. F. Gu, E. V. Pereloma, C. H. J. Davies. Mater. Sci. Eng. A. 492, $74-79$ (2008)

23. B. Oberdorfer et al. Acta Mater. 68, 189- 195 (2014)

24. A.P. Zhilyaev et al. Mater. Sci.Eng. A. 486, 123-126 (2008)

25. Y.S. Li, Y. Zhang, N.R. Tao, K.Lu. Acta Mater. 57, $761-772$ (2009)

26. B. Li, B. Y. Cao, K. T. Ramesh, E. Maa. Acta Mater. 57, 4500-4507 (2009) 